\title{
Evaluation of Wireless Body Area Networks
}

\author{
Kushnian Kour, Sandeep Singh Kang
}

\begin{abstract}
Remote body Area Network is the interconnection of different hubs that are situated in or around the outside of the body which is equipped for remote correspondence. Remote body Area Network includes different observing application condition, fighting, farming, military and social insurance. The principle motivation behind WBAN is to physiologically screen patient's fundamental signs and thus course the related information towards a base station. The sensor hubs are normally light weight, minimal effort, low power expending insight gadgets which are equipped for detecting, figuring, speaking with one another remotely. This audit gives an unmistakable outline about the elements of WBAN. The fundamental activity of conventions, transmitter and beneficiary of IEEE 802.15.6 are profoundly analyzed and examined in this work. The WBAN elements include the sensor devices.These sensor devises are wearable devices. These sensor devices are the detector devices such as motion sensors, ECG sensors etc. There are some protocols which are within body protocols in WBAN. These protocols enhance the work of the Wireless Body area network There are also some security aspects including confidentiality, integrity in WBAN. There are various application areas where these sensors can be used.This review paper gives snappy synopsis about the sensor plan, WBAN architecture, within-body routing protocols, applications, control proficiency, vitality preservation, correspondence conventions and security issues in WBAN.
\end{abstract}

Keywords-Wireless Body Area Networks (WBANs), Sensors, intra body and additional body correspondence, detector devices.

\section{INTRODUCTION}

In current innovation remote correspondence gives a ton of potential outcomes to have the capacity to share its data to one another at whenever and anyplace. Intelligent versatile correspondence system and WLAN, Wi-Fi are connected to different areas, for example, training; human services administration and industry so as to give individuals a helpful method to speak with one another. As the interest of omnipresent system is expanded, the devices for home, office and other data devices that can pass on remote in short range have been getting more thought. The standard and method improvement of pervasive system has quickly placed itself into the world market. Remote Body Area Network (WBAN) is turning into an extraordinary utilization of such method. WBAN varies with different remote sensor systems (WSN) with some critical focuses. First contrast between a WBAN and WSN is versatility. In WBAN client can move with sensor hubs with same portability design though WSN is commonly used to be stationary.

Revised Manuscript Received on July 22, 2019

Kushnian Kour, Dr. Sandeep Singh Kang ,University Institute of Engineering Department Computer Science Engineering Chandigarh University Mohali, Punjab (INDIA)
Vitality utilization is substantially less in WBAN than different WSNs arrangement. What's more WBAN sensor gadgets are discovered less expensive than WSNs. For unwavering quality, hub intricacy and thickness, WBAN hubs are anyway traditional. WSNs don't handle explicit prerequisites related with the communication between the system and the human body. A remote body territory organize (WBAN) associates autonomous hubs joined to the surface of body, embedded into tissues in body, or confined in home/human service applications, games, excitement, resistance, guileful registering and numerous different territories. WBAN does not compel the patient to keep on in the medical clinic along these lines giving much physical portability.

\section{LITERATURE REVIEW}

\section{A. WBAN INFRASTRUCTURE}

A WBAN comprises of in-body and on-body hubs that ceaselessly screen patient's fundamental data for analysis furthermore, medicine. Some on-body hubs can likewise be utilized for interactive media and gaming applications. A WBAN utilizes Remote Restorative Telemetry Administrations (WMTS), unlicensed Modern, Logical, and Therapeutic (ISM), Ultra-wideband (UWB), and Therapeutic Embed Correspondences Administration (MICS) groups for information transmission. WMTS is an authorized band utilized for therapeutic telemetry framework. Government Correspondence Commission (FCC) desires the utilization of WMTS for therapeutic applications due to less meddling sources. Nonetheless, just approved clients for example, doctors and prepared specialists are qualified to utilize this band. Besides, the confined WMTS (14 MHz) transfer speed can't bolster video and voice transmissions. The elective range for restorative applications is to utilize $2.4 \mathrm{GHz}$ ISM band that incorporates monitor groups to secure nearby channel impedance. An authorized MICS band (402-405 $\mathrm{MHz}$ ) is committed to the embed correspondence.[7]

\section{B. MAC PROTOCOLS}

The structure and usage of a low-control Macintosh convention for WBAN is at present a hot research subject. The most testing assignment is to oblige the in-body hubs in a power-productive way. Dissimilar to on-body hubs, the in-body hubs are embedded under human skin where the electrical properties of the body influence the sign proliferations. Li etal. proposed a novel TDMA convention for an on-body sensor arrange that misuses the bio signal highlights to perform TDMA synchronization and to improve the vitality proficiency. The presentation of a non-reference point IEEE 802.15.4 is researched, where the creators considered low transfer/download rates for the most part every hour. Be that as it may, it has no solid

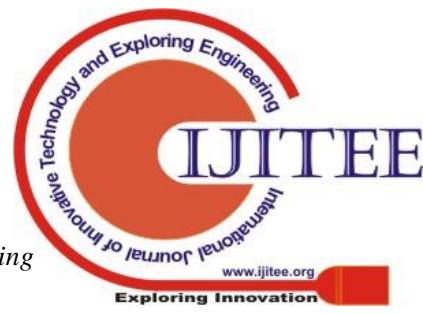


system characterized for on-request and crisis traffic.[11]

\section{IEEE 802.15.4}

The IEEE 802.15.4 can be considered for certain onbody sensor organize applications however it doesn't accomplish the required power dimension of in-body hubs. For basic and noncritical restorative traffic, the IEEE 802.15.4 has a few power utilization and QoS issues. Likewise, it works in 2.4 $\mathrm{GHz}$ band, which permits the conceivable outcomes of impedance from different gadgets, for example, IEEE 802.11 and microwave. Dave et al. examined the vitality proficiency and QoS execution of IEEE 802.15.4 and IEEE 802.11e.[11]

\section{WBAN ARCHITECTURE}

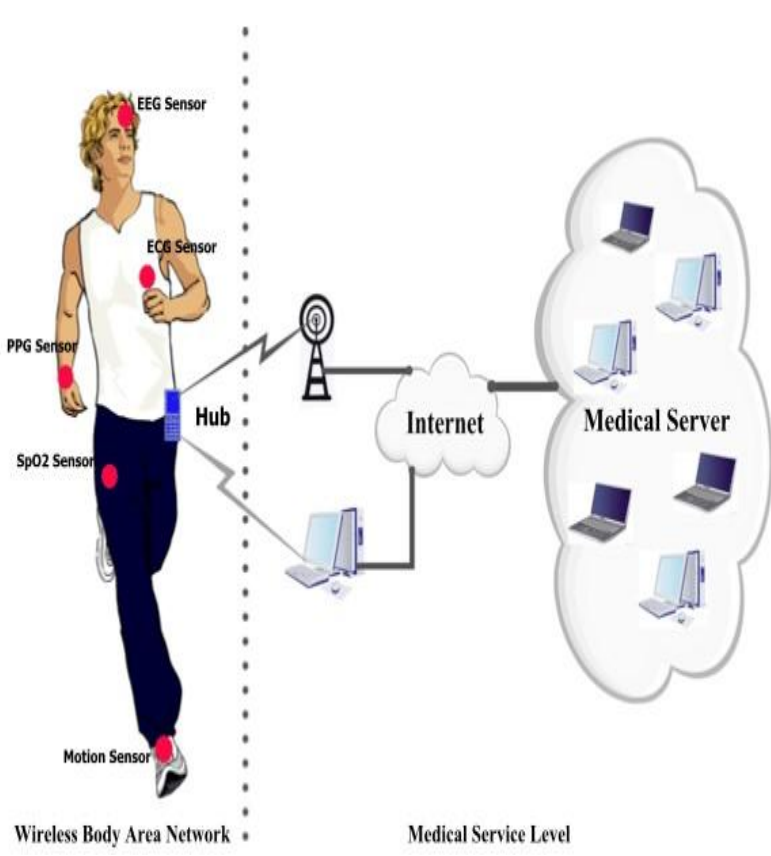

Fig 1:WBAN Architecture [9]

A WBAN architecture can be simply explained using two basic requirements, the human body and the medical server and their communication via internet. A human body is embedded with sensors including motion sensors ECG sensors and many other sensors. These sensors can be used to monitor the human medical conditions. These sensors sense the heart beat and other human activities and record them all. Through an internet medium all this data is transferred to the medical server. The medical server included the hospital ,ambulance, emergency services. If there is an emergency automatically an ambulance is send to patients place. The sensors are embedded in human body and a patient do not need to rest all the time he can move anytime anywhere. The Fig above shows the architecture of the wireless body area network

\section{WBAN APPLICATION AREAS}

The uses of Wireless Body Area Network(WBAN) are constrained. A great deal of advancement and research is going around there to enlarge its applications extend. WBAN discover its application for the most part in the restorative and medicinal services frameworks. These applications incorporate a few zones, for example, shrewd human services, helped old living, crisis reaction and intelligent gaming. Following are the fundamental uses of WBAN:

\section{A.TELEMEDICINE AND REMOTE PATIENT MOINTORING}

Telemedicine empowers the remote conveyance of patient consideration utilizing incorporated well being data frameworks and media transmission innovations and permits researchers, doctors and other medicinal experts around the globe to serve more patients. The total population add to the head ways in telemedicine arrange for the conveyance of a few human services administrations. Truth be told, on account of signs that body sensors provide, gathered data can be viably handled to get dependable and exact physiological estimations and to enable far off specialist to have continuous suppositions for therapeutic conclusion and medicine. Such savvy medicinal services framework can give applications to indicative method, support of unending condition and regulated recuperation from a careful procedure. Patient checking applications for the most part control imperative flags, and give ongoing input and data helping the recuperation of the patient. In such circumstance, we can hold the patient under specialist checking under regular physiological states without obliging their ordinary exercises and without harming him high cost. Daily-life movement checking screens the action amid day by day life of patients with some particular sicknesses; while in-medical clinic observing spotlights on cases in which patients need to remain in an emergency clinic for concentrated consideration and observations, sometimes for a delayed period. In the interim, a few therapeutic parameters are consistently recorded. Post-medical procedure in-home recuperation checking manages patients in their post recuperation period after a medicinal task/medical procedure and a recuperation period spent in emergency clinic. A WBAN can give constant estimations of the physiological parameters and permit better uncovering organ disappointments and quicker distinguishing crisis circumstances. Such remote checking framework will be more secure, increasingly advantageous and less expensive. In this field, numerous works have been proposed in writing. Some of them endeavoured to structure a conventional system ready to help most of cases, while others attempted to contemplate explicit diseases. Cardiovascular sicknesses, diabetes, malignant growth identification, asthma, Alzheimer and counterfeit retina are a few instances of explicit remote patient observing applications.[8]

\section{A. RECOVERY AND TREATMENT}

The objective of recovery is to enable patients to re establish their practical capacity to ordinary, through fitting rehabilitative medications after they were expelled from emergency clinic. Actually, restoration is a dynamic procedure which utilizes accessible offices to address any undesired movement conduct so as to achieve a desire .To empower an individual who has encountered a stroke to recapture the most noteworthy conceivable dimension of freedom with the goal that she can be as 
beneficial as could be allowed, the development of patients, in a recovery course, should be persistently observed and amended in order to hold a right movement design. Subsequently, identifying/following human development winds up crucial and vital in a locally established restoration conspire.

\section{B. BIOFEEDBACK}

Through WBAN, remote checking of human body should be possible. The information gathered by sensors can be gotten to assemble important parameters from the body. Patients can take care of and keep up their well being through the instrument of biofeedback like temperature investigation, pulse location, ECG, and so forth. Biofeedback implies keeping up and improving well being through gadgets that continually screens real exercises. This will make mindfulness with respect to certain physiological exercises.

\section{SURROUNDING ASSISTED LIVING}

The maturing populace, the expanding cost of formal social insurance and the significance that the people place on living autonomously, all spur the advancement of creative helped living innovations for protected and free maturing. Applications in this field improve personal satisfaction so as to keep up a progressively autonomous way of life utilizing home computerization. Truth be told, helped living offices have risen as an elective lodging office for individuals with inabilities and older who are not viewed as free but rather don't require nonstop therapeutic consideration, as in nursing or retirement homes. An surrounding sensor system can detect and control the parameters of the living condition and after that conveys the body information to a focal station, on account of a consistent psychological and physical observing. The well being state of these individuals can be evaluated from their heart beat rate, circulatory strain and accelerometer information. The framework perhaps associated with a social insurance community for perception and crisis help, if there should arise an occurrence of solid changes in the watched parameters or deviations from the ordinary range.

\section{WAY OF LIFE AND SPORTS}

Wireless Body Area Network is using to determine the heading the club of golf and the branch of the golfer to offer continuous reaction to the golfer as far as big development, pet subtleties on the fairway. The melioration and the influence of the golf club can likewise be fearless. I gathering, the organism tactile information of a competitor's development is made to inspect, monitor vitality and influence the competitor to perform at the greatest elevation period of time. Body Area Networks support new capacities with regards to remote body-driven frameworks including wearable incitement structure (e.g., music fervor), coordinating help in the vehicle or while walking, presentation corridor, heartbeat and execution seeing in amusements, preschooler checking, remote cash card.

\section{ARMED APPLICATIONS}

The Research Laboratory of Army (ARL) has achieve tests utilizing sound-related sensor clusters hanging underneath fastened aerostats to detect and bind temporary signs out of missile, and armaments. This persistent review can possess a persuasive result on the durability and deadlines of our officers. Rousing the exhibit gives an apparently greater scope of location beneath complimenting MET conditions. The open doors for utilizing BANs in the powers are various. A portion of the military application for BANs incorporates observing wellbeing, area. An uniform for fight contained with a body area network may turn into a suitable computerized system that associates gadgets, for example, life bolster detectors, camcorders, RF and individual PDAs, wellbeing observing, and transfer information to and from the trooper's suitable PC. The system could perform capacities, for example, concoction acknowledgment, discovery to keep unfortunate casualties from agreeable flame and checking of a fighter's anatomical state. Line for help, his radio sends and gets signals with a receiving wire mixed into his uniform. Thus, BANs present new open doors for war zone lethality and survivability.

\section{POSITIONING WBANS}

The conventions created for WBANs can length from correspondence between the body sensors to correspondence from a body hub to a server farm associated with the web. In this manner correspondence in WBAN is separated into:

A. Intra body communication

B. Extra body communication

Figure below shows positioning of wbans diagrammatically.

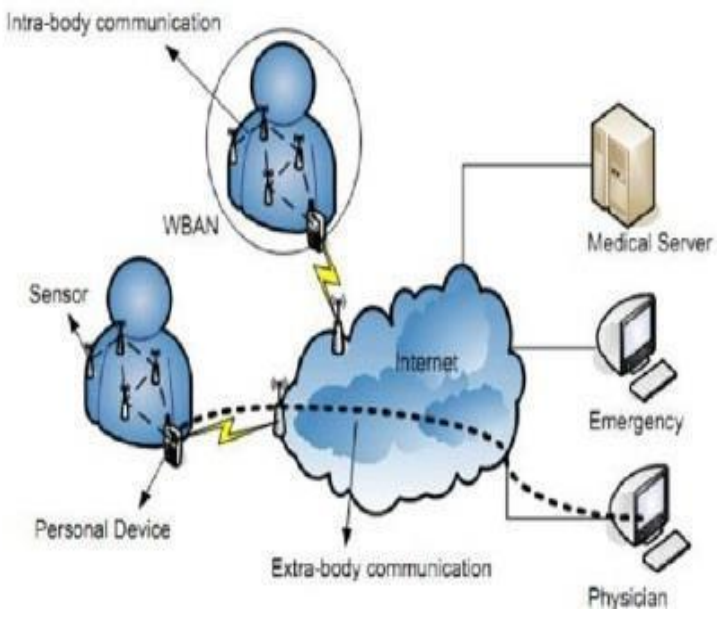

Fig 2: Positioning of WBANS [5]

Intra body and additional body correspondence: Intra body correspondence controls the data dealing with on the body between the sensors or actuators and individual gadget. Correspondence between the individual gadgets and an outside network. This division is like the one characterized in where a multi-layered telemedicine framework is introduced. Level 1incorporates the intra-body correspondence, level 2 the additional body correspondence between the individual gadgets and the Internet and level 3 speaks to the additional body correspondence from the web to the medicinal server. 


\section{DETECTOR DEVICES}

Detectors are the key segments of a wireless body area network, as they connect the visible world and computerized systems. For the most part, they can be ordered within actinic, warm, machine-driven, and sensual detectors. Past examinations possess showed that the density and plenty fullness scope of human physical signals are similarly little; thus, a little inspecting recurrence and little information transmission rate would be adequate. Be that as it may, what kind of and what number of sensors a wireless body area network system employs depend generally on the application scenario and the framework foundation. To better monitor a human's imperative signs, conduct, and surrounding condition, a wide scope of commercially accessible sensors can be conveyed, such as accelerometer and whirligig, , electromyography, and electroencephalography cathodes, beat oximetry, respiration, carbon dioxide blood pres-beyond any doubt, glucose, stickiness, in addition to detectors. Often utilized detector devices for wireless body area networks are overviewed beneath.

Acceleration sensors are generally utilized for movement arrest. As an expansion or option to an accelerometer, a spinner can be utilized for applications, for example, mobile step monitor in gand examination. For recognizing movements, an accelerometer/spinner cluster with several sensors can be conveyed. This brings up issues for positioning and clamor decrease techniques .Proper situating diminishes the quantity of accelerometers/whirligigs required and the resulting information rate. Diminishing the quantity of sensors decreases the movement location motion to-commotion proportion because of lower excess, consequently requiring the sensors to be conveyed at the planned locations with higher exactness.

Figure below shows Body area sensor network node architecture.

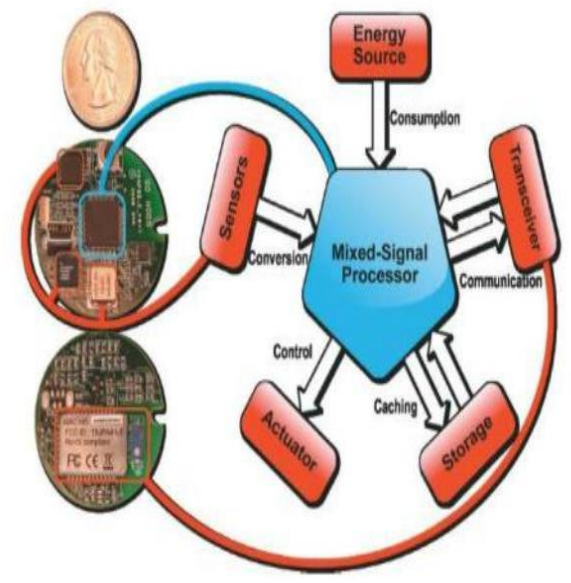

Fig 3: BASN node architecture [6]

\section{WITHIN BODY ROUTING PROTOCOLS IN WBAN}

Considering they are planned to work within the human body, the following directing conventions were structured in a manner to regard the warm prerequisites of biomedical applications where the scarcest ascent in temperature can prop bacterial development or cause extensive harms to the encompassing tissues.

\section{A. THERMAL-AWARE ROUTING ALGORITHM(TARA)}

In Tang et al. presented TARA, the first steering convention to feature the issue of temperature ascend in warm touchy applications. TARA works in two stages, a setup stage and a directing stage. In the arrangement stage, each hub of the system screens its bystanders by watching their exercises so as to get an estimation of their heat changes dependent on the sent bundle check. This progression permits finding hotspots (regions where the heat of sensor hubs surpasses a predefined limit). The estimation is at that point recorded on an inner rundown that will be traded with experienced hubs. In the directing stage, bundles are sent to the goal by applying a withdrawal procedure that comprises of straying hotspots by discovering elective courses. Assuming no elective course is to be discovered, the parcel is cushioned for a certain time so as to allow the hotspot to cool down, on the off chance that the time terminates before the chilling off, at that point the parcel is dropped.[7]

\section{B. LEAST TEMPERATURE ROUTING(LTR)}

LTR (Least Temperature Routing) introduced in is a warm mindful directing convention dependent on TARA. In LTR, as in TARA, every hub of the system screens its neighbours by watching their exercises so as to get an estimation of their temperature changes dependent on the sent parcel check. In the sending stage, LTR courses parcels to the goal through the "coolest hubs", the hubs with the least temperature among the neighboring hubs. Data about the as of late visited hubs is kept in each bundle in request to abstain from coming back to the last hub simply because it is the coolest one, In which case, the bundle is sent to the "following coolest hub". LTR abstains from circling by determining a Max-Hop incentive to the bundle which on the off chance that it is surpassed, the parcel will be dropped.

Since LTR utilizes the "coolest hubs" to advance the bundles, a low most extreme temperature rise is guaranteed. In any case, a great deal of hubs are probably going to be utilized before achieving the sink hub which may prompt a high by and large system temperature and real power utilization. What's more, since the course determination doesn't consider the separation between the source and goal hubs, the parcel isn't ensured to utilize the ideal one, which means a high conveyance delay.

\section{THERMAL-AWARE SHORTEST HOP ROUTING(TSHR)}

The warm mindful most limited bounce steering, or TSHR, is a convention bound to biomedical applications with organized bundle conveyance limitations that permits the re transmission of dropped bundles. This convention characterizes two sorts of limits. A foreordained edge TS, fixed for all hubs that, determines the most extreme temperature a hub can't surpass. Also, a powerfully produced limit called TDN in light of the neighboring hubs' and the hub's own temperature. This limit is utilized to distinguish a hotspot. In truth, a hub is viewed as a 
hotspot when its temperature outperforms TDN.

TSHR performs in two stages: a setup stage, where every hub constructs its steering table, and a directing stage where information parcels are steered through the briefest way. At the point when a hub needs to forward a parcel, the following jump hub's temperature is contrasted with TS, in the event that the temperature is more prominent than TS, at that point the bundle is cradled until the temperature chills off. In the other hand, if the following bounce hub is a hotspot (reasoned from TDN), at that point another "coolest" hub must be chosen to forward the information bundle.

When contrasted with HPR and SHR, TSHR has a high conveyance delay in high parcel entry rate, yet guarantees lower temperature rise and no bundle drops. TSHR is additionally appeared give the most elevated system lifetime.

\section{NEW ENERGY-EFFICIENT ROUTING PROTOCOL(M-ATTEMPT)}

This convention named M-ATTEPT was intended for heterogeneous bio-medicinal sensor systems with vitality productivity and defer requirements. The design used to speak to the proposed convention comprises of sensor hubs arranged on various pieces of the human body. The sink hub was fixed on the focal point of the body while different hubs were set on the less versatile parts as indicated by their information rates. This convention maintains a strategic distance from the warming effects of the biomedical sensor hubs on the human body by utilizing a system utilized by hubs closer to the sink (high information rate hubs). On the off chance that these hubs temperature achieves a specific limit, they break the connection that associates them to their neighboring hubs for few rounds until their temperature chills off.

M-ATTEMPT, isolates information bundles into three classes:

Basic information parcels, inquiry driven information bundles and standard information parcels. Simply after all the basic and the inquiry driven information parcels have been transmitted to the sink hub in an one hop manner by expanding the information transmission rate, standard information parcels are sent by the sensor hubs Sin a multi-jump design through the way with the less bounce tally. MATTEMPT works in four stages. In the introduction stage, neighbor hubs' and sink hubs' position data (in terms of jump check) is communicated by means of a welcome messages.

\section{E. ADAPTIVE LEAST TEMPERATURE ROUTING} (ALTR)

This convention named ALTR, for Adaptive Least Temperature Directing, works similarly LTR does when it comes to sending the parcel to the coolest neighboring hub and not surpassing a jump check esteem, here called "Versatile Hop-Count". What varies from LTR is the manner by which ALTR handles the sent bundles which bounce check outperforms the predefined esteem. Without a doubt, not at all like in LTR, these bundles will not be dropped. Rather, ALTR applies a Shortest Hop Calculation (SHA) that courses the bundle through the most limited way regardless of how hot the included hubs are. In the other hand, to adjust the utilization of hotspots in specific cases, this convention sets a "proactive postponement "instrument that forfeits the parcel conveyance postponement to diminish the temperature ascend by giving the sent bundles, that are going to a generally high temperature "coolest hub", a postponement of one time unit before it is sent to the "following coolest hub". In ALTR, the conveyance defer diminishes when the most brief way is utilized. Be that as it may, as an exchange off, it might cause temperature rise on the off chance that the most brief way incorporates hotspots. Since ALTR utilizes least temperature hubs to advance a parcel, which may take a moderately lengthy time-frame before touching base to the goal. Thusly an ascent in the general system temperature and control utilization is likely.

\section{F. HOTSPOT PREVENTING ROUTIN(HPR)}

\section{In Bag et al., proposed Hotspot Preventing Routing}

(HPR) calculation, a convention expected to limit the normal parcel conveyance postpone together with avoiding hotspot arrangement in sensor biomedical systems. HPR works in degrees; an arrangement stage and a guidance degree. inside the arrangement stage, a steerage desk is labored at each hub depending on the records traded about introductory temperatures and the most restrained way. in the steerage degree, whilst no hotspot is to be experienced, the maximum brief way calculation is utilized to deliver the bundles. Contrariwise, while the maximum quick way experiences a hotspot, the sensor hub endeavours to evade it by way of sending the package deal to a non as of late visited friendly hub with the least temperature (coolest bystander). so one can make experience of whilst a bystander hub has simply been visited, in which case the parcel is despatched to the subsequent coolest bystander hub, each sensor hub keeps a rundown of the hesitantly visited hubs.

A friendly hub is perceived to experience a hotspot, if its heat is more elevated than the aggregate of the hub's very own temperature and an edge. The limit esteem is set powerfully dependent on the nearby burden and the heat of the friendly hubs. Like in LTR, HPR utilizes a Max-Hop Count to drop bundles that surpass the quantity of jumps allowed.

Despite the fact that the recreation results have demonstrated TARA to beat HPR at high bundle entry rates, it has in the other hand demonstrated that HPR plays out the best as far as low heat rise, parcel misfortune and bundle conveyance delays.

\section{CHALLENGES OF WBAN}

In spite of the fact that WBAN is helping significantly in medicinal and social insurance frameworks, still there are difficulties which are to be handled.

\section{A. SECURITY}

Security is the primary test for WBAN. Endeavors are being made to make information transmissions in WBAN progressively secure and exact. An individual patient's information ought not blend with other patient's information. The information created in the system ought to be secure so that there is no unapproved access to the information. Secrecy, trustworthiness, and verification are the principle security prerequisites of WBAN. 


\section{B. INTEROPERABILITY}

The information exchange ought to be smooth crosswise over different innovation models like Bluetooth, ZigBee. The WBAN framework ought to likewise be adaptable with the end goal that the system availability isn't hindered when the system is broadened.

\section{PRIVACY}

Individuals are anxious about the possibility that that their private data may get spilled while utilizing the WBAN arrange. In this way WBAN must guarantee the protection and security of the individual data.

\section{INFORMATION CONSISTENCY}

The consistency of information ought to be kept up. In the remote body region organize, the information is divided and exchanged at different hubs. This can cause irregularity of information. In this manner consistency of information ought to be kept up.

\section{E. IMPEDANCE}

For the substantial scale usage of WBAN framework, impedance ought to be less. This will expand the conjunction between the sensor hubs with different gadgets in the system.

\section{PROTECTION IN WBAN}

Before impel secret writing, the protection procedure focus on in the lie-down location. Moreover, for multi modal frameworks, for example, RFID improved obtrusive social insurance structure, the security matter for each methodology ought to be intentional incoherently and there must be intense instruments against a wide range of assaults. Remote Body Area Systems (WBAN) has given to help progressively wellbeing observing of a patient and analyze numerous life dangers ailments. It works inside a human and bolsters therapeutic and non-therapeutic applications. This formal shows a review of the protection highlights of the wireless body area network framework that was organized in The institute of Electrical and Electronics Engineers 802.15.6 standard. This characterizes the 3 dimensions of security. Every security level has its very own security properties. These territories,

Position 1 this is unbound method of correspondence. This level has no system of examining of validation and affirmation.

Position 2 this is the dimension of Institute of Electrical and Electronics Engineers in which validation is examined as it were. In this dimension information isn't encoded.

Position 3 this is the dimension of Institute of Electrical and Electronics Engineers in which verification and secret writing are examined; it is huge amount of protection.

In future position 3 will be embraced in wireless body area network for protection and protection dangers. In wireless body area network protection is the basic matter, so for this reason recurrence circle determination, chamber displaying, radio wire plan, physical convention structure, vitality productive equipment, MAC convention plan, QoS and unwavering quality, continuous availability, systems, administrative consistence, are given significance. The main protection issues are:

\section{A. INFORMATION CONFIDENTIALITY INTEGRITY}

Information privacy is viewed as the most imperative issue in WBANs. It is utilized to ensure the information and maintain a strategic distance from spilling out the information. Information respectability is likewise imperative in perspective on life basic occasions of patient. It might be unsafe. At the point when crisis information of any patient is changed, information might be lost because of awful correspondence condition.[2]

\section{B. INFORMATION AUTHENTICATION}

It checks the first source code. On the off chance that it disregards information confirmation then information bundle may endure. It very well may be accomplished by Message Authentication Code (MAC) that is for the most part registered from the mutual mystery key.

\section{INFORMATION FRESHNESS AND STABLE LOCALIZATION}

In this protected information, information is refreshed again and again. It's not possible for anyone to take outdated information and answer outdated ideas. There are 2 kinds of information freshness: feeble freshness, which give fractional information to the edges in an arrangement however may some postponement, and in solid freshness, information outlines are sent rapidly and there is no deferral. In wireless body area network applications it is important to locate the precise area of sufferer. In this method following instrument is connected [2]

\section{ACCESSIBITY AND SECURE MANAGEMENT}

Fast reaction of sufferer to the doctor is also essential, so the doctor recommend needed prescription or different safety measures. On the off chance that this is moderate life might be lost. One of the most ideal ways is to switch the activity of a hub that has been assaulted to another hub in the system. Secure administration is additionally important to arrange the gave key dispersion to the hubs of the information in secret writing and unscrambling structure.

\section{CONCLUSION}

This paper gives an expansive audit of wireless body area network, its application zones, dares and protection issues. Wireless body area network can be utilized to recognize character, crisis, medicinal, military, remote observing, and gathering data. In wireless body area network the fundamental difficulties are its safety, protection, adaptability and responsibility. Wireless body area networks are the arrangement of proper safety and preservation insurance for the remote correspondence medial. The information going midst the sensors hubs ought to kept secret and honesty ensured. WBAN is a framework that gives smooth more affordable and walking review amid routine capacities works in close relationship with remote body zone organize. It additionally gives better and modest substitutions for accomplishing great wellbeing conditions. These frameworks decrease the colossal expenses related to sufferer in medical clinics as observing continuously even at home and over a more drawn out period. Along these lines an

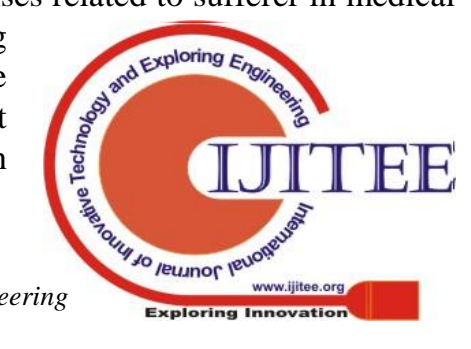


extraordinary advantage goes to patients, doctors just as the entire society. The objective of this audit formal isn't to censure the current wireless body area recovery frameworks, in any case fill in as an inference for prevailing achievement and to give course for future investigation growth.

\section{REFERENCES}

1. Mark A. Hanson, Harry C. Powell Jr., AdamT. Barth, Kyle Ringgen berg, Benton H. Calhoun, James H.Aylor, and John Lach Body Area Sensor Networks: Challenges and Opportunities , University of Virginia, IEEE Computer Society (2009)

2. Data Security AndPrivacy In Wireless Body Area Networks, Ming L And Wenjing Lou, Worcester Polytechnic Institute Kui Ren, Illinois Institute Of Technology, IEEE Wireless Communications ( February 2010)

3. https://www.seminarsonly.com/computer\%20science/wireless-body-are a-network.php

4. https://www.techsparks.co.in/thesis-in-wireless-body-area-network/

5. https://www.researchgate.net/figure/Example-of-intra-body-and-extra-b ody-communication-in-a-WBAN_fig24_45709672

6. https://www3.nd.edu/ dwang5/courses/spring19/ papers/bsn/BSNOverview.pdf

7. Javaid, N., Abbas, Z., Fareed, M.S., Khan, Z.A. and Alrajeh, N., 2013 M-ATTEMPT: A new energy-efficient routing protocol for wireless body area sensor networks. Procedia Computer Science, 19, pp.224-231.
8. Ragesh, G. K., \& Baskaran, K. (2012). An Overview of Applications , Standards and Challenges in Futuristic Wireless Body Area Networks. Journal of Computer Science

9. Transmission-Rate-Adaption Assisted Energy-Efficient Resource Allocation With QoS Support in WBANs

10. Tang, Q.,Tummala, N., Gupta, S.K. and Schwiebert, L., 2005, June. TARA: thermal-aware routing algorithm for implanted sensor networks.International Conference on Distributed Computing in Sensor Systems (pp. 206-217). Springer Berlin Heidelberg

11. https://www.researchgate.net/publication/253418115 IEEE 802154 _for_Wireless_Sensor_Networks_A_Technical_Overview

\section{AUTHORS PROFILE}

Kushnian kour, Research Scholar, University Institute of Engineering Department Computer Science Engineering Chandigarh University Mohali, Punjab (INDIA) BE:YEAR 2011-2015 MBSCET -JAMMU UNIVERSITY Kushniankour123456@gmail.com

Dr. Sandeep Singh Kang professor, university institute of engineering department computer science engineering chandigarh university mohali, punjab

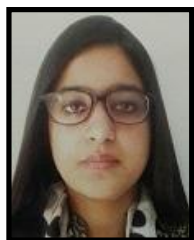
(india) education: JNV punjabi university. his area of research is wireless networks. email: sandeepkang.cse@cumail.in 